\title{
Tension dynamics in semiflexible polymers. I. Coarse-grained equations of motion
}

\author{
Oskar Hallatschek, ${ }^{1, *}$ Erwin Frey, ${ }^{2}$ and Klaus Kroy $^{3}$ \\ ${ }^{1}$ Lyman Laboratory of Physics, Harvard University, Cambridge, Massachusetts 02138, USA \\ ${ }^{2}$ Arnold Sommerfeld Center for Theoretical Physics and Center for NanoScience, LMU München, Theresienstrasse 37, 800333 München, \\ Germany \\ ${ }^{3}$ Institut für Theoretische Physik, Universität Leipzig, Augustusplatz 10/11, 04109 Leipzig, Germany
}

(Received 27 September 2006; published 7 March 2007)

\begin{abstract}
Based on the wormlike chain model, a coarse-grained description of the nonlinear dynamics of a weakly bending semiflexible polymer is developed. By means of a multiple-scale perturbation analysis, a length-scale separation inherent to the weakly bending limit is exploited to reveal the deterministic nature of the spatio temporal relaxation of the backbone tension and to deduce the corresponding coarse-grained equation of motion. From this partial integro-differential equation, some detailed analytical predictions for the nonlinear response of a weakly bending polymer are derived in an accompanying paper [O. Hallatschek et al., following paper, Phys. Rev. E 75, 031906 (2007)].
\end{abstract}

DOI: 10.1103/PhysRevE.75.031905

PACS number(s): 87.15.He, 87.15.Aa, 87.16.Ka, 83.10.-y

\section{INTRODUCTION}

The laws of Brownian motion have played the role of a mediator between the apparently smooth deterministic dynamics on a macroscopic scale and the microscopic molecular chaos since their discovery a century ago [1]. They are pivotal to our understanding of a broad class of animate and inanimate soft condensed-matter systems that owe their characteristic softness to low-dimensional and strongly fluctuating mesoscale structures such as polymeric networks and membranes [2]. Conversely, these systems are well suited to study how complex deterministic dynamics on a macroscale or mesoscale emerges from the underlying stochastic differential equations [3].

Take, for example, a stiff polymer like actin that is suddenly stretched by strong forces applied at its ends. Or, conversely, consider a polymer that is held in a virtually straight conformation and suppose that the forces at its ends are suddenly released. These two paradigmatic experimental setups, which we call pulling and release, are illustrated in Fig. 1. How will the end-to-end distance of the polymer relax to its new equilibrium value? Given the manifestly stochastic un-

*Electronic address: ohallats@fas.harvard.edu

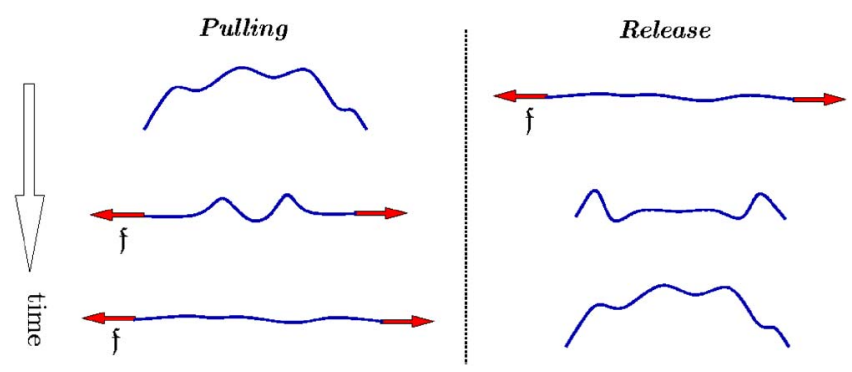

FIG. 1. (Color online) Two basic examples of dynamic forceextension experiments. Pulling: a weakly bending polymer in equilibrium is suddenly pulled longitudinally by two external forces $\mathfrak{f}$. Release: a prestretched polymer is suddenly released. derlying dynamics, which for release is exclusively driven by thermal forces, it is not immediately obvious that the initial contraction or stretching dynamics should obey a deterministic law, as tacitly assumed by several heuristic derivations [4-8]. Indeed, these studies, which predicted a variety of interesting new dynamic scaling regimes, arrived at partially contradicting results $[4,7]$. Despite considerable experimental, theoretical, and numerical work, both relaxation laws (for pulling and release) remained controversial for some time.

It therefore appears worthwhile to undertake a detailed mathematical derivation of the mesoscale dynamic equations that govern the nonlinear dynamics of semiflexible polymers "from first principles" [3] — i.e., from the underlying stochastic differential equations of motion. In a recent Letter, we outlined such a systematic approach that resolves the aforementioned theoretical problems, together with some of its consequences [9]. The present contribution offers a more comprehensive discussion. In this paper, part I, an effective coarse-grained mesoscale description of the dynamics of a semiflexible polymer is developed by means of a multiplescale theory from the stochastic differential equations of motion. Our detailed analysis also reveals the limits of validity of the deterministic mesoscopic description and shows how to deal with subtle end effects that may in some cases mask the nontrivial predictions for certain observables. Building on this general framework, the theory is elaborated for the specific problems of pulling and release in part II [10], which provides a template for the future analysis of a variety of related problems with somewhat different boundary and initial conditions [11-13]. Thereby, we corroborate the importance of a regime of homogeneous tension relaxation, which generally occurs in release experiments and establish the complete crossover scenario between the various intermediate scaling regimes. Additionally, in part II some consequences for common observables are worked out in detail to facilitate experimental verification of the theory.

Before entering a detailed quantitative analysis, it seems useful to summarize the main ideas on a qualitative level in order to make the remainder more easily accessible. A characteristic property of semiflexible polymers and many other 
fluctuating mesoscale structures in soft condensed matter is their reduced dimensionality or slender shape. It entails the presence of thermally excited transverse fluctuations of an essentially inextensible backbone. Returning to the above example, an actin filament is much more susceptible to bending undulations than to stretching or compressing its backbone. An analogous statement holds for other biopolymers or twodimensional locally flat objects like membranes and surfaces. This suggests to idealize these structures as undulating inextensible manifolds [14]. To be specific, we focus for the following on the case of a single semiflexible polymer in solution, which seems to be the simplest paradigmatic example of the more general soft-matter mesostructures alluded to above. Its equilibrium mechanical properties and conformational statistics have by now been thoroughly studied theoretically and experimentally $[15,16]$. Both are well understood in terms of the self-affine roughness of the equilibrium contour fluctuations within the so-called wormlike chain model, which idealizes the polymer as an inextensible space curve with an energetic cost for bending [17]. Here, we are primarily interested in the transient nonequilibrium stretching and contraction dynamics of such a wormlike chaini.e., in the much less studied problem of how a semiflexible polymer relaxes to equilibrium after a sudden drastic change in its boundary conditions. This question is of considerable fundamental and practical interest alike-e.g., for singlemolecule manipulations [18-20] and for understanding and controlling the dynamic response of polymer solutions and networks such as those constituting the cytoskeleton of biological cells $[21,22]$.

Because of the inextensible backbone, the stretching or contraction dynamics of a wormlike chain is entirely due to a spatiotemporal reorganization of the contour length stored in the transverse thermal wrinkles. It is governed by the dynamic backbone tension $f(s, t)$, which is the force that holds the backbone together. The dynamics is always assumed to be strongly overdamped by solvent friction, which can be decomposed into transverse and longitudinal components with respect to the local tangent, in view of the locally rodlike conformation of the polymer. At first sight, one might suppose that for small enough undulations one can resort to a formulation of the dynamics solely in terms of transverse modes for which only transverse friction matters. Actually, for the equilibrium dynamics in the absence of external forces, the conclusions based on such an assumption are in accordance with a series of experimental data [23-26]. At second thought, considering the constraint of inextensibility, it is far from obvious how the interplay between transverse and longitudinal friction limits the relaxation after a sudden application or release of external forces such as, e.g., in release and pulling. It is the major objective of the present study to resolve this puzzle for the case of longitudinal forces, while the somewhat more complex issue of the nonlinear transverse response will be addressed in a separate contribution [13].

As a cornerstone of our derivation, we establish a dynamic scale separation between the scales where transverse and longitudinal friction reign: namely, the transverse and longitudinal dynamic correlation lengths $\ell_{\perp}(t)$ and $\ell_{\|}(t)$, respectively. We demonstrate that $\ell_{\perp}(t) \ll \ell_{\|}(t)$ holds at any time in the limit of a weakly bending rod. As a central result, we obtain that the tension varies to leading order only in the large scale $\ell_{\|}(t)$, over which the short-wavelength transverse undulations that dominate the dissipation on the scale $\ell_{\perp}(t)$ are self-averaging. The short-scale transverse fluctuations may thus be said to provide an effective local backbone compliance for the large-scale longitudinal dynamics. In this way, transverse and longitudinal dynamics are effectively decoupled, and the microscopic stochastic differential equations can be reduced, in a controlled way, to a deterministic (integro-differential) equation for the coarse-grained mesoscale dynamics.

With some care, most of the predictions that emanate from this reduced description can qualitatively be obtained from a relatively simple scaling analysis, to which we will occasionally resort in order to promote the intuitive physical understanding of our analysis. In fact, not only physical insights but also several interesting formal predictions were originally obtained on the basis of simple scaling arguments. However, due to some subtleties and pitfalls, the results available in the literature remained somewhat contradictory. The following development, in particular part I of this article series, aims at settling the corresponding issues conclusively by employing a controlled perturbation theory instead of evoking scaling assumptions.

As suggested by the introductory examples of dynamic force-extension experiments, exemplary realizations of the mesoscale dynamics occur in response to highly localized forces, which we generally (though not invariably) assume to be applied abruptly at the boundaries. They initiate universal self-similar relaxation processes that spread through the polymer, resulting in characteristic power-law signatures, socalled intermediate asymptotics [27], in various observables. These will be derived analytically in part II [10], which is devoted to solving the effective deterministic mesoscale equations for the tension, obtained below. There, we will also consider the consequences of the tension dynamics on pertinent observables like the (projected) end-to-end distance and the novel experimental perspectives brought up by our analysis. For the impatient reader, our Letter [9] may serve as a quick guide to our main arguments and results.

The outline of the present part (part I) is as follows. We develop the systematic coarse-grained description of tension dynamics in stiff and stretched semiflexible polymers, respectively, that emerges from an appropriate small gradient expansion of the dynamical wormlike chain model (Sec. II). An ordinary perturbation expansion leading to linear firstorder equations of motion (Sec. III) turns out to be restricted to times when the tension has already relaxed to its equilibrium value (Sec. IV). The actual tension dynamics on shorter times is resolved by a stochastic multiple-scale perturbation theory (MSPT) (Sec. V), which is based on a dynamical length-scale separation. As a major result, we obtain a rigorous deterministic partial integro-differential equation (PIDE) that describes the long-wavelength (all-time) dynamics of the tension.

\section{DYNAMICAL WORMLIKE CHAIN MODEL}

At low Reynolds numbers, the dynamics of a polymer is determined by the balance of elastic forces, friction, and sto- 
chastic forces. We will briefly motivate how these forces are modeled in the usual stochastic description of the Brownian motion of a semiflexible polymer, leading to Eq. (11) below, which is the basis of our subsequent analysis.

The natural model for the description of the elastic properties of a semiflexible polymer arises from the idea to regard the polymer as a thin cylinder consisting of a homogeneous elastic material. In the slender limit, where the ratio of thickness over total length approaches zero, the deformation modes of the cylinder become much stiffer in the axial direction ("phonons") than in directions transverse to the cylinder axis ("bending modes") [28]. Consequently, such a slender rod or "thread" subject to external forces merely allows for bending deformations, while its contour length is to a good approximation locally conserved.

These features are idealized in the wormlike chain model, where the polymer is at any time $t$ represented as an inextensible space curve $\boldsymbol{r}(s, t)$ parametrized by the arc length $s$; i.e., the tangents have to obey the constraint

$$
\boldsymbol{r}^{\prime 2}=1 .
$$

Here, we have introduced the shorthand notation $\boldsymbol{r}^{\prime}$ $\equiv \partial \boldsymbol{r}(s, t) / \partial s$. The effective free energy $\mathcal{H}_{\mathrm{WLC}}$ of a particular conformation is only due to bending (curvature) and is given by

$$
\mathcal{H}_{\mathrm{WLC}}=\frac{\kappa}{2} \int_{0}^{L} d s \boldsymbol{r}^{\prime 2},
$$

where $\kappa$ is the bending stiffness. To assure that the integrand is the square of the local curvature, the inextensibility constraint, Eq. (1), has to be enforced as a rigid constraint.

The elastic force (per arclength) $\boldsymbol{g}_{\mathrm{el}}$ derives from Eq. (2) by a functional derivative,

$$
\boldsymbol{g}_{\mathrm{el}}(s, t)=-\left.\frac{\delta \mathcal{H}_{\mathrm{WLC}}}{\delta \boldsymbol{r}}\right|_{\boldsymbol{r}^{\prime 2}=1} .
$$

As indicated, the contour variations $\delta r$ used to determine the functional derivative on the right-hand side have to respect the local inextensibility constraint, Eq. (1). The variational calculation, detailed in Appendix A, yields

$$
\boldsymbol{r}^{\prime} \times\left(\kappa \boldsymbol{r}^{\prime \prime \prime}+f_{\mathrm{el}}\right)=\mathbf{0},
$$

where $f_{\mathrm{el}}(s) \equiv \int_{0}^{s} d \widetilde{s} \boldsymbol{g}_{\mathrm{el}}(\widetilde{s})$ is the spatial integral over the elastic force density defined in Eq. (3).

According to the implicit equation for $f_{\mathrm{el}}$, Eq. (4), the force $\kappa \boldsymbol{r}^{\prime \prime \prime}+f_{\text {el }}$ has a vanishing component transverse to the local tangent $\boldsymbol{r}^{\prime}$. Equivalently, we may require that both vectors are proportional to each other,

$$
\kappa \boldsymbol{r}^{\prime \prime \prime}+f_{\mathrm{el}}=f \boldsymbol{r}^{\prime},
$$

where the proportionality factor $f(s)$ has dimensions of a force and, in general, depends on the arclength. A spatial derivative then yields a direct expression for the elastic force density,

$$
g_{\mathrm{el}}=-\kappa \boldsymbol{r}^{\prime \prime \prime \prime}+\left(f \boldsymbol{r}^{\prime}\right)^{\prime} .
$$

The apparent simplification with respect to Eq. (4) is somewhat deceiving, since Eq. (6) still contains the unknown function $f(s)$, which has to be fixed by the local arclength constraint, Eq. (1). The new force field $f(s)$ has, however, a very direct and intuitive physical interpretation. It is the local line tension [29] that "ties the polymer together" and thereby enforces the inextensibility condition.

Much of the following deals with the dynamics of this local line tension, which turns out to be closely related to the dynamics of the local excess length stored in undulations, defined in Eq. (18) below, to which we will refer to as the stored length. Time dependence is introduced into the description by requiring the elastic force density $\boldsymbol{g}_{\text {el }}$ to be balanced by the dynamic friction (per arclength) with the solvent,

$$
\boldsymbol{g}_{\mathrm{fr}}(s, t)=-\boldsymbol{\zeta}(\boldsymbol{r}, t) \partial_{t} \boldsymbol{r}(s, t) \quad \text { (free draining). }
$$

At any arclength $s$ and time $t$, the friction matrix $\zeta$ with elements $\zeta_{i j}$ can be decomposed into its transverse and longitudinal components with respect to the local tangent $\boldsymbol{r}^{\prime}(s, t)$,

$$
\boldsymbol{\zeta}=\left[\zeta_{\perp}\left(1-\boldsymbol{r}^{\prime} \otimes \boldsymbol{r}^{\prime}\right)+\zeta_{\|} \boldsymbol{r}^{\prime} \otimes \boldsymbol{r}^{\prime}\right] .
$$

The constants $\zeta_{\perp}$ and $\zeta_{\|}$can to a first approximation be estimated by the friction coefficients (per length) for transverse and longitudinal motion, respectively, of a rigid slender rod in a solvent of viscosity $\eta[30]$,

$$
\zeta_{\perp}=2 \zeta_{\|} \simeq 4 \pi \eta .
$$

A more sophisticated analysis would consider logarithmic corrections [31-33] to account for the dynamic coupling of distant chain segments $s$ and $s^{\prime}$ via long-ranged hydrodynamic interactions $[30,34]$. While such logarithmic factors may sometimes be crucial in a quantitative comparison with some experiments $[23,26]$, their (feasible) implementation is not of primary interest to our present discussion, so that we chose to dismiss them for greater clarity of the presentation.

On the same level of approximation, the force balance of elastic and frictional forces can be extended by adding thermal white noise fully characterized in terms of its mean and variance,

$$
\begin{gathered}
\left\langle\xi_{i}(s, t)\right\rangle=0, \\
\left\langle\xi_{i}(s, t) \xi_{j}\left(s^{\prime}, t^{\prime}\right)\right\rangle=2 k_{B} T \zeta_{i j} \delta\left(s-s^{\prime}\right) \delta\left(t-t^{\prime}\right),
\end{gathered}
$$

with the angular brackets indicating an ensemble average. The strength of the noise correlations in Eq. (10b) is dictated by the fluctuation-dissipation theorem $[35,36]$, which assures that the steady state of the stochastic equations of motion corresponds to thermal equilibrium (for complications in simulations of discrete bead-rod chains, see Refs. [37-39]).

Upon using Eqs. (6) and (7), the balance of elastic, friction, and stochastic forces, $0=\boldsymbol{g}_{\mathrm{el}}+\boldsymbol{g}_{\mathrm{fr}}+\boldsymbol{\xi}$, takes the form of an equation of motion, 


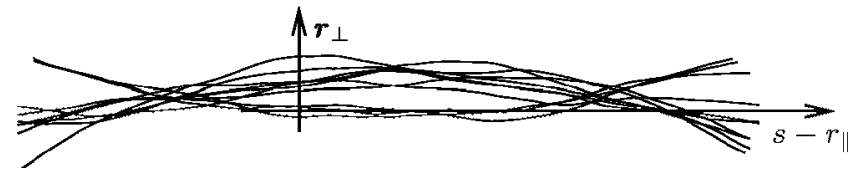

FIG. 2. Typical conformations of a freely fluctuating stiff polymer with suppressed rotations. In order to generate these conformations, which serve illustrative purposes only, we have represented each conformation as a linear superposition of the first ten modes that solve the linearized equation of motion. For each realization, the mode amplitudes were then drawn randomly according to their Gaussian distribution in equilibrium.

$$
\boldsymbol{\zeta} \partial_{t} \boldsymbol{r}=-\kappa \boldsymbol{r}^{\prime \prime \prime \prime}+\left(f \boldsymbol{r}^{\prime}\right)^{\prime}+\boldsymbol{\xi}
$$

The partial differential equation (11), the arclength constraint, Eq. (1), and the Gaussian noise-correlation comprise a complete stochastic description ${ }^{1}$ of the Brownian dynamics in the free draining limit.

\section{LINEARIZED STOCHASTIC DYNAMICS}

The nonlinear stochastic dynamics of a wormlike chain, represented by Eq. (11), in combination with the inextensibility constraint, Eq. (1), is hard to analyze in general. The difficulties are largely due to the calculation of the line tension $f(s, t)$, which has to enforce the local inextensibility constraint, Eq. (1). There have been attempts to relax the local constraint and merely enforce local inextensibility on average [40-42]. This corresponds to replacing the field $f(s, t)$ by a (spatially averaged) mean field. However, those approaches fail to describe semiflexible polymers on local scales, where tension fluctuations (in time and space) are important [4].

Our analytical approach is instead based on the weakly bending limit, in which the polymer conformation is appropriately described by its deviations from a straight line. Accordingly, we parametrize the polymer's space curve by

$$
\boldsymbol{r}=\left(\boldsymbol{r}_{\perp}, s-r_{\|}\right)^{T}
$$

(cf. Fig. 2), where $\boldsymbol{r}_{\perp}(s)$ and $r_{\|}(s)$ are the transverse and longitudinal displacements at arclength $s$. Such parametrization is only useful if the gradients of the transverse displacements are small everywhere along the contour,

$$
{\boldsymbol{r}_{\perp}^{\prime}}^{2}=O(\epsilon) \ll 1, \quad s \in(0, L) .
$$

The weakly bending limit, as defined in Eq. (12), assumes that there is a small parameter $\epsilon$ that controls the polymer roughness uniformly along the contour. In the case of a stiff polymer, on which we focus unless otherwise stated, the small parameter is provided by the ratio

$$
\epsilon \equiv \frac{L}{\ell_{p}}=\frac{L k_{B} T}{\kappa} \ll 1 \quad \text { (stiff polymer) }
$$

of length $L$ over persistence length $\ell_{p}$. Alternatively, a weakly bending conformation may be realized by applying a

\footnotetext{
${ }^{1}$ Since the thermal forces have a physical origin, Eq. (11) has to be interpreted according to Stratonovič [50].
}

static external stretching force $f$ larger than the internal characteristic force scale $k_{B} T / \ell_{p}$. In this case, $r_{\perp}^{\prime 2} \sim \sqrt{k_{B} T / \ell_{p} f}$ [43] and we may identify

$$
\epsilon \equiv \sqrt{k_{B} T / \ell_{p} \mathfrak{f}} \quad \text { (stretched polymer) }
$$

Equation (12) allows us to expand the dynamical equations of motion in terms of small gradients. We start with the inextensibility constraint, which "enslaves" the higher-order longitudinal displacements to the transverse ones. After resolving Eq. (1) for $r_{\|}^{\prime}$ and expanding the square root, the local constraint takes the simple form

$$
r_{\|}^{\prime}=\frac{1}{2} \boldsymbol{r}_{\perp}^{\prime 2}+O\left(\epsilon^{2}\right)=O(\epsilon)
$$

This entails that the parameter $\epsilon$ is a measure for the reduction of the longitudinal extension

$$
R_{\|} \equiv L-r_{\|}(L)+r_{\|}(0)
$$

of the polymer due to the presence of thermal undulations: An arclength integral of Eq. (15) shows that $R_{\|}$is smaller then the total contour length $L$ by an amount of the order

$$
L-R_{\|}=r_{\|}(L)-r_{\|}(0)=O(\epsilon L) .
$$

We may think of the length difference in Eq. (17) as being "stored" in undulations. The distribution of this excess length along the filament is, according to Eq. (15), described by the function

$$
\varrho(s, t) \equiv \frac{1}{2} \boldsymbol{r}_{\perp}^{\prime 2}(s, t)=O(\epsilon),
$$

which will have central importance in our analysis. It is the fraction of the contour length that is at arclength $s$ and time $t$ locally stored in undulations.

After taking a "spatial" (i.e., arclength) derivative of its longitudinal part, we expand the equation of motion, Eq. (11), to order $O\left({r_{\perp}^{\prime}}^{2}\right)=O(\epsilon)$ and obtain

$$
\begin{gathered}
\partial_{t} \boldsymbol{r}_{\perp}=-\boldsymbol{r}_{\perp}^{\prime \prime \prime \prime}+\left(f \boldsymbol{r}_{\perp}^{\prime}\right)^{\prime}+\mathfrak{g}_{\perp}+\boldsymbol{\xi}_{\perp} \\
\hat{\zeta} \partial_{t} r_{\|}^{\prime}=(\hat{\zeta}-1)\left(\boldsymbol{r}_{\perp}^{\prime} \partial_{t} \boldsymbol{r}_{\perp}\right)^{\prime}-r_{\|}^{\prime \prime \prime \prime \prime}-f^{\prime \prime}+\left(f r_{\|}^{\prime}\right)^{\prime \prime}-\mathfrak{g}_{\|}^{\prime}-\xi_{\|}^{\prime} .
\end{gathered}
$$

Here, we have neglected terms of order $O\left(\epsilon^{3 / 2}\right)$ and made the following choice of units: time and tension, respectively, are rescaled according to

$$
\begin{gathered}
t \rightarrow \zeta_{\perp} t / \kappa, \\
f \rightarrow \kappa f .
\end{gathered}
$$

This corresponds to setting $\kappa \equiv \zeta_{\perp} \equiv 1$ and $\hat{\zeta} \equiv 1 / 2=\zeta_{\|}$. As a consequence all variables represent powers of length; e.g., $t$ and $f$ are a length ${ }^{4}$ and a length ${ }^{-2}$, respectively. In Eq. (19) we have further allowed for an external ${ }^{2}$ force density $\mathfrak{g}$ $=\left(\mathfrak{g}_{\perp}, \mathfrak{g}_{\|}\right)$, which is a length ${ }^{-3}$, that may, for instance, repre-

\footnotetext{
${ }^{2}$ Throughout, we reserve Gothic font for external forces or force fields like $\mathfrak{g}$.
} 
sent the effect of a solvent flow, an optical-magnetical tweezer, or an electrical field.

As long as one is taking the limit $\epsilon \rightarrow 0$ with $t, s$, and $L$ fixed, the polymer is correctly described by the linearized version of Eq. (19),

$$
\begin{gathered}
\partial_{t} \boldsymbol{r}_{\perp}=-\boldsymbol{r}_{\perp}^{\prime \prime \prime \prime}+\left(f \boldsymbol{r}_{\perp}^{\prime}\right)^{\prime}+\mathfrak{g}_{\perp}+\boldsymbol{\xi}_{\perp}, \\
f^{\prime \prime}=-\mathfrak{g}_{\|}^{\prime} .
\end{gathered}
$$

From the magnitude of the noise correlations, given by Eq. (10b), it may be inferred that $\boldsymbol{\xi}_{\perp}$ to leading order obeys

$$
\left\langle\boldsymbol{\xi}_{\perp}(s, t) \boldsymbol{\xi}_{\perp}\left(s^{\prime}, t^{\prime}\right)\right\rangle=2\left(\mathbf{I} / \ell_{p}\right) \delta\left(s-s^{\prime}\right) \delta\left(t-t^{\prime}\right),
$$

where we used $k_{B} T \zeta_{\perp} \rightarrow \ell_{p}^{-1}$ in our choice of units and $\mathbf{I}_{i j}$ $=\delta_{i j}$ is the identity matrix.

From Eq. (22b), it is seen that the tension profile of a polymer that is forced at the ends is linear; slope and offset are fixed by the boundary conditions. The (higher-order) longitudinal displacements are enslaved to the transverse ones by the arclength constraint, Eq. (15). At the present level of approximation the exact equations of motion have reduced to a linear equation for the transverse displacements alone.

\section{A. Generalized transverse response}

In many practical cases-for instance, if the polymer is symmetrically pulled apart by a (possibly time-dependent) force-the tension $f=f(t)$ is to lowest order spatially homogeneous such that the equation of motion, Eq. (22a), reduces to

$$
\partial_{t} \boldsymbol{r}_{\perp}=-\boldsymbol{r}_{\perp}^{\prime \prime \prime \prime}+f \boldsymbol{r}_{\perp}^{\prime \prime}+\boldsymbol{\xi}_{\perp} .
$$

Let us anticipate at this point that we will identify an inherent length-scale separation, Eq. (58) in Sec. V below, according to which the tension can be considered as slowly varying in space, such that Eq. (24) describes the polymer dynamics locally (and even globally at late times). As a consequence, the solution of Eq. (24) for a given spatially homogeneous tension history $f(t)$ becomes an important ingredient of the nonlinear theory and shall be analyzed in the following.

Formally, the linear Langevin equation (25) is solved by

$$
\boldsymbol{r}_{\perp}(s, t)=\int_{0}^{L} d s^{\prime} \int_{-\infty}^{\infty} d t^{\prime} \chi\left(s, s^{\prime} ; t, t^{\prime}\right) \boldsymbol{\xi}_{\perp}\left(s^{\prime}, t^{\prime}\right) .
$$

The Green's function $\chi\left(s, s^{\prime} ; t, t^{\prime}\right)$ satisfies

$$
\begin{aligned}
\partial_{t} \chi\left(s, s^{\prime} ; t, t^{\prime}\right)= & -\partial_{s}^{4} \chi\left(s, s^{\prime} ; t, t^{\prime}\right)+f(t) \partial_{s}^{2} \chi\left(s, s^{\prime} ; t, t^{\prime}\right)+\delta(s \\
& \left.-s^{\prime}\right) \delta\left(t-t^{\prime}\right)
\end{aligned}
$$

and appropriate boundary conditions. It may be interpreted as a causal response function that describes the spreading and the decay of contour undulations induced by a transverse force impulse at location $s^{\prime}$ and (elapsed) time $t^{\prime}$. Equation (25) therefore can be said to represent the conformation at time $t$ in terms of the accumulated response to the transverse noise history $\boldsymbol{\xi}_{\perp}\left(s^{\prime}, t^{\prime}\right)$ along the contour.

In the general case of a time-dependent tension, it can be quite difficult to determine the Green's function $\chi$ that obeys the prescribed boundary conditions, because eigenmodes and eigenvalues of the linear operator $\left[f(t)+\partial_{s}^{2}\right] \partial_{s}^{2}$ depend on the value of $f(t)$ and thus become time dependent. In terms of Fourier modes, on the other hand, a translationally invariant Green's function $\bar{\chi}\left(s-s^{\prime} ; t, t^{\prime}\right)$ can easily be found (see below). As will be detailed in Sec. IV, this function describes the universal bulk dynamics far away from the ends, while a correction term that manifestly breaks translational invariance has to be added "close" to the ends to correct for the actual boundary effects.

To formalize this decomposition into bulk and boundaries, the full response function $\chi$ may be written as a superposition

$$
\chi\left(s, s^{\prime} ; t, t^{\prime}\right)=\bar{\chi}\left(s-s^{\prime} ; t, t^{\prime}\right)+\chi_{b c}\left(s, s^{\prime} ; t, t^{\prime}\right),
$$

where $\bar{\chi}\left(s-s^{\prime} ; t, t^{\prime}\right)$ and $\chi_{b c}\left(s, s^{\prime} ; t, t^{\prime}\right)$ represent a translationally invariant part and the boundary correction, respectively. The former is taken to satisfy

$$
\partial_{t} \bar{\chi}\left(s ; t, t^{\prime}\right)=\left[-\partial_{s}^{2}+f(t)\right] \partial_{s}^{2} \bar{\chi}\left(s ; t, t^{\prime}\right)+\delta(s) \delta\left(t-t^{\prime}\right)
$$

on an infinite arclength interval. With help of Fourier modes

$$
\bar{\chi}\left(q ; t, t^{\prime}\right) \equiv \int_{-\infty}^{\infty} d s \bar{\chi}\left(s ; t, t^{\prime}\right) e^{-i q s},
$$

Eq. (28) reads

$$
\partial_{t} \bar{\chi}\left(q ; t, t^{\prime}\right)+\lambda(q, t) \bar{\chi}\left(q ; t, t^{\prime}\right)=\delta\left(t-t^{\prime}\right),
$$

where $\lambda(q, t)$ is the dispersion relation ${ }^{3}$

$$
\lambda(q, t)=q^{4}+f(t) q^{2} .
$$

By the method of integrated factors, the solution to Eq. (30) is found to be ${ }^{4}$

$$
\bar{\chi}\left(q ; t, t^{\prime}\right)=\Theta\left(t-t^{\prime}\right) \exp \left[-\int_{t^{\prime}}^{t} d \hat{t} \lambda(q, \hat{t})\right],
$$

which may be checked by direct substitution. The real-space susceptibility is given by the inverse Fourier transform of Eq. (32),

$$
\bar{\chi}\left(s ; t, t^{\prime}\right)=\int_{0}^{\infty} \frac{d q}{\pi} \bar{\chi}\left(q ; t, t^{\prime}\right) \cos (q s),
$$

where it has been used that $\bar{\chi}\left(q ; t, t^{\prime}\right)$ is even in $q$.

The part $\chi_{b c}\left(s, s^{\prime} ; t, t^{\prime}\right)$ of the susceptibility, which is not translationally invariant, satisfies the homogeneous differential equation

\footnotetext{
${ }^{3}$ The dispersion relation, Eq. (31), is easily extended to accommodate a general time-dependent transverse harmonic confinement potential represented by an additional spring constant on the righthand side of Eq. (31).

${ }^{4}$ We note aside that $\bar{\chi}^{2}(q ; t, 0)$ is identical to the "amplification factor" introduced in Ref. [47] to describe the growth of the squared-mode amplitudes.
} 


$$
\partial_{t} \chi_{b c}=\left[-\partial_{s}^{2}+f(t)\right] \partial_{s}^{2} \chi_{b c}
$$

and has boundary conditions that have to compensate for the generally inappropriate behavior of $\bar{\chi}\left(s-s^{\prime} ; t, t^{\prime}\right)$ at the boundaries. The difficulty of solving Eq. (26) has been shifted to $\chi_{b c}\left(s, s^{\prime} ; t, t^{\prime}\right)$. However, for the time-dependent quantities to be studied below, this boundary term represents a relevant contribution only within a characteristic length $\ell_{\perp}(t)$ (defined below) close to the boundaries. For times small enough, such that $\ell_{\perp}(t) \ll L$, one may use $\chi_{b c}$ derived on a semi-infinite polymer to approximate the situation near one boundary - say, the one at $s=0$. For simplicity, we will mostly refer to the model boundary conditions of "hinged" (h) or "clamped" (c) ends [44], for which the full susceptibility on a semi-infinite arclength interval $s \in(0, \infty)$ is given by a symmetric and antisymmetric combination of the bulk susceptibility $\bar{\chi}$,

$$
\chi_{h / c}\left(s, s^{\prime} ; t, t^{\prime}\right)=\bar{\chi}\left(s-s^{\prime} ; t, t^{\prime}\right) \mp \bar{\chi}\left(s+s^{\prime} ; t, t^{\prime}\right) .
$$

Evidently, $\chi_{h / c}$ satisfies Eq. (26) on $s \in(0, \infty)$, as well as the boundary conditions

$$
\begin{gathered}
\chi_{h}\left(0, s^{\prime} ; t, t^{\prime}\right)=0=\partial_{s}^{2} \chi_{h}\left(0, s^{\prime} ; t, t^{\prime}\right) \quad \text { (hinged) }, \\
\partial_{s} \chi_{c}\left(0, s^{\prime} ; t, t^{\prime}\right)=0=\partial_{s}^{3} \chi_{c}\left(0, s^{\prime} ; t, t^{\prime}\right) \quad \text { (clamped). }
\end{gathered}
$$

A hinged end has vanishing transverse displacement and is torque free (vanishing second derivative), whereas a ("gliding") clamped end has a vanishing slope and is force free (vanishing third derivative).

\section{B. Local response of the stored excess length}

As a basis for our subsequent systematic analysis of tension dynamics and as an application of the above results, we wish to determine the longitudinal motion implied by the linearized transverse stochastic dynamics, Eq. (22a). To this end, consider a stiff polymer equilibrated under a constant tension $f_{<}$at time zero, on which a spatially constant tension $f(t)$ is imposed that varies deterministically for $t>0$. We ask how the density of stored excess length $\varrho(s, t)$, defined in Eq. (18), changes in time by considering the ensemble average of the increase (during the time interval $t$ ) of the stored length,

$$
\Delta \varrho(s, t) \equiv \varrho(s, t)-\varrho(s, 0)
$$

This is an important observable, since it governs the leadingorder contribution to the change

$$
\Delta R_{\|}(t) \equiv R_{\|}(t)-R_{\|}(0)
$$

in the projected end-to-end distance $R_{\|}(t)$, defined in Eq. (16), which is can be directly measured in dynamic singlepolymer experiments [23]. Here, the (average) end-to-end axis of the polymer is assumed to be controlled by external means-e.g., by an external force field, flow field, boundary conditions, etc. To the relevant order, the precise measures taken to orient the polymer (strictly or on average) do not matter, and we find

$$
\left\langle\Delta R_{\|}\right\rangle(t)=-\int_{0}^{L} d s\langle\Delta \varrho\rangle(s, t)+o(\epsilon)
$$

Here, the notation with the arguments $s$ and $t$ outside the brackets of $\langle\Delta \varrho\rangle$ was introduced to emphasize that, even after averaging, these dependences generally persist.

For an explicit calculation of the stored length, we insert Eq. (25) into Eq. (18) and perform an ensemble average upon employing Eq. (23):

$$
\langle\varrho\rangle(s, t)=\int_{-\infty}^{t} d t^{\prime} \int_{0}^{L} d s^{\prime} \int_{-\infty}^{t} d \widetilde{t}^{\prime} \int_{0}^{L} d \widetilde{s}^{\prime} \partial_{s} \chi\left(s, s^{\prime} ; t, t^{\prime}\right) \partial_{s} \chi\left(s, \widetilde{s}^{\prime} ; t, \widetilde{t}^{\prime}\right)\left\langle\boldsymbol{\xi}_{\perp}\left(s^{\prime}, t^{\prime}\right) \cdot \boldsymbol{\xi}_{\perp}\left(\widetilde{s}^{\prime}, \widetilde{t}^{\prime}\right)\right\rangle / 2=2 \int_{-\infty}^{t} \frac{d t^{\prime}}{\ell_{p}\left(t^{\prime}\right)} \int_{0}^{L} d s^{\prime} \partial_{s} \chi\left(s, s^{\prime} ; t, t^{\prime}\right)^{2}
$$

For later convenience, we have allowed for a time-dependent persistence length $\ell_{p}(t)$ and an optional prestress $f_{<} \gg L^{2}$. The latter is also technically advantageous, since it acts as a physical regularization to suppress modes with wavelength larger than the total length. It enables us to take the total length to infinity and to discuss the stored length $\langle\varrho\rangle(s, t)$ on a semi-infinite arclength interval. For our ultimate goal of calculating $\langle\Delta \varrho\rangle(s, t)$ an intrinsic regularization renders modes with wavelength beyond a characteristic length scale $\ell_{\perp}(t)$ irrelevant, so that $f_{<}$can eventually be set to zero if required.

Inserting Eq. (35), valid for hinged and clamped boundary conditions, into Eq. (38) (with $L \rightarrow \infty$ ) yields

$$
\begin{gathered}
\left\langle\Delta \varrho_{h / c}\right\rangle=\int_{0}^{\infty} \frac{d q}{\pi}[\hat{\varrho}(q, t)-\hat{\varrho}(q, 0)][1 \pm \cos (2 q s)], \\
\hat{\varrho}(q, t)=2 q^{2} \int_{-\infty}^{t} d \widetilde{t} \bar{\chi}^{2}(q ; t, \widetilde{t}) / \ell_{p}(\widetilde{t}) .
\end{gathered}
$$

The general expression, Eq. (39), is now specialized to the scenario 


$$
\begin{aligned}
& \text { tension persistence length } \\
& t<0: \quad f_{<}=\text {const, } \ell_{p}=\text { const, } \\
& t>0: \quad f(t), \quad \ell_{p} / \theta=\text { const. }
\end{aligned}
$$

As preparation for a more general discussion, we have included the possibility of a sudden change in persistence length by a factor $1 / \theta>0$ at $t=0$.

With a constant tension at negative times, the time integral in Eq. (39) can be evaluated from $\tilde{t}=-\infty$ to $\tilde{t}=0$, after which we obtain

$$
\hat{\varrho}(q, t) \ell_{p}=\frac{\bar{\chi}^{2}(q ; t, 0)}{q^{2}+f_{<}}+2 \theta q^{2} \int_{0}^{t} d \widetilde{t}^{2}(q ; t, \widetilde{t})
$$

Therewith, the full expression for the average change in stored excess length density becomes

$$
\begin{aligned}
\left\langle\Delta \varrho_{h / c}\right\rangle= & \int_{0}^{\infty} \frac{d q}{\pi \ell_{p}}\left\{\frac{1}{q^{2}+f_{<}}\left(e^{-2 q^{2}\left[q^{2} t+F(t)\right]}-1\right)\right. \\
& \left.+2 \theta q^{2} \int_{0}^{t} d \widetilde{t} e^{-2 q^{2}\left[q^{2}(t-\widetilde{t})+F(t)-F(\tilde{t})\right]}\right\}[1 \pm \cos (2 q s)],
\end{aligned}
$$

where $F(t)$ is the integral of the tension over positive times,

$$
F(t)=\int_{0}^{t} d \hat{t} f(\hat{t})
$$

One observes that the integral in Eq. (42) is well defined, even for $f_{<}=0$, because the integrand vanishes for $q \rightarrow 0, \infty$. It is dominated by wave numbers for which the exponents become of order 1; i.e., the dominant modes have wave numbers $q$ for which the characteristic relaxation time

$$
\tau_{q}=\left(q^{4}+q^{2} F / t\right)^{-1}
$$

is of the order of $t$. This suggests to define a characteristic length $\ell_{\perp}(t)$ as the wavelength for which the relaxation time is just $t$,

$$
1=\ell_{\perp}^{-2}\left[\ell_{\perp}^{-2} t+F(t)\right]
$$

Asymptotically $\ell_{\perp}(t)$ is given by

$$
\ell_{\perp}(t) \sim \begin{cases}t^{1 / 4}, & \text { for } t \ll(F / t)^{-2}, \\ F^{1 / 2}, & \text { for } t \gg(F / t)^{-2} .\end{cases}
$$

Due to the competition between bending forces ( $\left.\propto r_{\perp} \ell_{\perp}^{-4}\right)$ and tension $\left(\propto r_{\perp}(F / t) \ell_{\perp}^{-2}\right)$, the growth of $\ell_{\perp}$ thus exhibits a dynamic crossover from free relaxation $\left(\ell_{\perp}\right.$ $\left.\simeq t^{1 / 4}\right)$ to relaxation under tension $\left(\ell_{\perp} \simeq \sqrt{F}\right)$ at a characteristic time $t_{\mathrm{f}} \equiv(F / t)^{-2}$ (see Table I, left, for a constant tension equal to the external force $\mathfrak{f}$ ).
TABLE I. The transverse equilibration length $\ell_{\perp}(t)$ and the tension propagation length $\ell_{\|}(t)$ both exhibit a crossover at a time $t_{f}$ $\equiv \mathfrak{f}^{-2}$, which depends on the external force $\mathfrak{f}$ (here, for the pulling problem with $\mathfrak{f} \gg L^{-2}$ ).

\begin{tabular}{lcc}
\hline \hline & $\ell_{\perp}(t)$ & $\ell_{\|}(t)$ \\
\hline$t \ll t_{\mathfrak{f}}$ & $t^{1 / 4}$ & $t^{1 / 8}\left(\ell_{p} / \zeta\right)^{1 / 2}[6,8]$ \\
$t \gg t_{\mathfrak{f}}$ & $t^{1 / 2} \mathfrak{f}^{1 / 2}$ & $t^{1 / 4} \mathfrak{f}^{1 / 4}\left(\ell_{p} / \zeta\right)^{1 / 2}[4]$ \\
\hline
\end{tabular}

As we will explicitely demonstrate in the next section, the change $\left\langle\Delta \varrho_{h / c}\right\rangle$ in stored length saturates at a constant value for distances to the boundaries much larger than the characteristic length $\ell_{\perp}(t)$. This "bulk" value is given by ${ }^{5}$

$$
\begin{aligned}
\langle\Delta \bar{\varrho}\rangle(t) \equiv & \int_{0}^{\infty} \frac{d q}{\pi \ell_{p}}\left\{\frac{1}{q^{2}+f_{<}}\left(e^{-2 q^{2}\left[q^{2} t+F(t)\right]}-1\right)\right. \\
& \left.+2 \theta q^{2} \int_{0}^{t} d \widetilde{t} e^{-2 q^{2}\left[q^{2}(t-\widetilde{t})+F(t)-F(\tilde{t})\right]}\right\} .
\end{aligned}
$$

The quantity $\langle\Delta \bar{\varrho}\rangle(t)$ will be central in our systematic analysis of tension propagation and relaxation, because it turns out to determine the local curvature of the tension profile. Each of the two terms inside the curly brackets of Eq. (47) have a direct physical interpretation. Since the parameter $\theta$ tunes the strength of the thermal kicks, it is seen that the $\theta$-independent first term represents the deterministic change in the excess length that is stored in mode $q$ in absence of any stochastic force. For pulling forces $F>0$ its sign is always negative, since both the internal elastic and the external driving forces act to straighten the filament. On the contrary, thermal kicks represented by the (strictly positive) second term favor undulations.

\section{BREAKDOWN OF ORDINARY PERTURBATION THEORY}

The previous sections employed "ordinary" perturbation theory (OPT) in the small parameter $\epsilon$, leading to a linear equation of motion to lowest order. As detailed below, the use of OPT is, however, limited to long times even for $\epsilon \ll 1$. The predictions derived above for the longitudinal segment motion turn out to be incompatible with the longitudinal force balance on short times. In particular, Eq. (47) reveals an infinite longitudinal friction for $t \rightarrow 0$. This section extends a heuristic argument of Ref. [6] to resolve this problem. The following, furthermore, elucidates a very general feature of the nonlinear response: namely, a crossover from "weak"- to "strong"-force behavior. Finally, it reveals the crucial length-scale separation underlying our subsequent systematic analysis.

The breakdown of OPT becomes evident when we try to use Eq. (42) to evaluate the longitudinal segment motion in a

\footnotetext{
${ }^{5}$ This equation for the stored length at a spatially constant but time-dependent tension has recently independently been derived in a related context [51].
} 
nonequilibrium situation. For definiteness and as a telling example, let us consider an initially equilibrated polymer that is suddenly pulled longitudinally by a constant force $f$ at both ends; i.e., at time $t=0$, the tension at the ends is suddenly increased from 0 to a given positive value $\mathfrak{f}$,

$$
\mathfrak{f}=f(0, t>0)=f(L, t>0) \quad \text { (pulling scenario). }
$$

As a consequence of Eq. (22b), the tension $f=\mathfrak{f} \Theta(t)$ is to lowest order spatially uniform and fixed by the driving force $f$ at the boundaries. Our above result for the change in stored length due to a given tension history, Eq. (42), thus applies and evaluates to

$$
\begin{aligned}
\left\langle\Delta \varrho_{h / c}\right\rangle= & \int_{0}^{\infty} \frac{d q}{\pi \ell_{p}} \frac{\mathfrak{f}}{q^{2}\left(q^{2}+\mathfrak{f}\right)}\left[e^{-2 q^{2}\left(q^{2}+\mathfrak{f}\right) t}-1\right] \\
& \times[1 \pm \cos (2 q s)] .
\end{aligned}
$$

After the variable substitutions $k \equiv q \mathfrak{f}^{-1 / 2}, \sigma \equiv s \mathfrak{f}^{1 / 2}$, and $\tau$ $\equiv \mathfrak{f}^{2} t=t / t_{\mathfrak{f}}$, Eq. (48) takes the form

$$
\left\langle\Delta \varrho_{h / c}\right\rangle=\ell_{p}^{-1} \mathfrak{f}^{-1 / 2} \Sigma_{h / c}(\sigma, \tau)
$$

with the scaling function

$$
\Sigma_{h / c}(\sigma, \tau)=\int_{0}^{\infty} \frac{d k}{\pi} \frac{1}{k^{2}\left(k^{2}+1\right)}\left[e^{-2 k^{2}\left(k^{2}+1\right) \tau}-1\right][1 \pm \cos (2 k \sigma)] .
$$

Another variable substitution $\widetilde{k}=k \tau^{1 / 4}$ generates factors $\widetilde{k}^{4}$ $+\widetilde{k}^{2} \tau^{1 / 2}$ in the exponent and denominator, which can be replaced by $\widetilde{k}^{4}$ in the asymptotic limit $\tau \ll 1$. Just as for the dispersion relation, Eq. (44), tensile forces $\propto k^{2}$ may be neglected as compared to the dominant bending forces $\propto k^{4}$ for times smaller than the crossover time $t_{\mathrm{f}}$. In the opposite limit $\tau \gg 1$, the reverse approximation applies. After a variable substitution $\tilde{k}=k \tau^{1 / 2}$, factors $\widetilde{k}^{4} \tau^{-1}+\widetilde{k}^{2}$ appear, which may be replaced by $\tilde{k}^{2}$. We thus find that the two-parameter scaling form, Eq. (50), collapses onto one-parameter scaling forms for small and large times,

$$
\begin{array}{ll}
\Sigma_{h / c} \sim-\tau^{3 / 4} \Sigma_{h / c}^{<}\left(\sigma \tau^{-1 / 4}\right), & \tau \rightarrow 0, \\
\Sigma_{h / c} \sim-\tau^{1 / 2} \Sigma_{h / c}^{>}\left(\sigma \tau^{-1 / 2}\right), & \tau \rightarrow \infty,
\end{array}
$$

with scaling functions given by

$$
\begin{aligned}
& \Sigma_{h / c}^{<}(\xi)=\int_{0}^{\infty} \frac{d \widetilde{k}}{\pi} \frac{1-e^{-2 \tilde{k}^{4}}}{\widetilde{k}^{4}}[1 \pm \cos (2 \tilde{k} \xi)], \\
& \Sigma_{h / c}^{>}(\xi)=\int_{0}^{\infty} \frac{d \widetilde{k}}{\pi} \frac{1-e^{-2 \tilde{k}^{2}}}{\widetilde{k}^{2}}[1 \pm \cos (2 \tilde{k} \xi)] .
\end{aligned}
$$

Note that the spatial part of these scaling functions depicted in Fig. 3 decays to zero within several rescaled time units. As an important consequence, we note that the part of $\left\langle\Delta \varrho_{h / c}\right\rangle$ that depends on the boundary conditions really only matters close to the boundaries-i.e., up to a distance for which the scaling variable becomes of order 1 . In fact, this distance can

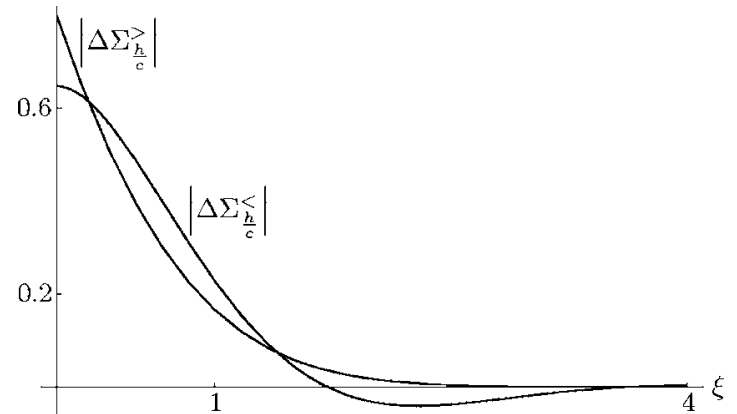

FIG. 3. The absolute value $\left|\Delta \Sigma_{h / c}^{\gtrless}\right|$ of the spatially varying part $\Delta \Sigma_{h / c}^{\gtrless} \equiv \Sigma_{h / c}^{\gtrless}(\xi)-\Sigma_{h / c}^{\gtrless}(\infty)$ of the scaling functions $\Sigma_{h / c}^{\gtrless}$ defined in Eqs. (51) and (52), which happens to be the same for hinged and clamped boundary conditions.

be identified with $\ell_{\perp}(t)$, as defined in Eq. (45), because the scaling variable of $\Sigma$ is given by $\sigma \tau^{-1 / 4}=s / t^{1 / 4}=s / \ell_{\perp}(t)$ for $\tau \ll 1$ and $\sigma \tau^{-1 / 2}=s / \ell_{\perp}(t)$ for $\tau \gg 1$, respectively.

The bulk of the polymer stores length according to the universal part $\langle\Delta \bar{\varrho}\rangle(t)$ (independent of the boundary condition), which asymptotically takes the form

$$
\langle\Delta \bar{\varrho}\rangle(t) \sim\left\{\begin{array}{lr}
\Gamma(7 / 4)^{-1} \mathfrak{f} \ell_{p}^{-1}(t / 2)^{3 / 4}[45], & \text { for } t \ll t_{\mathfrak{f}}, \\
\ell_{p}^{-1}(2 \mathfrak{f} t / \pi)^{1 / 2}, & \text { for } t \gg t_{\mathfrak{f}} .
\end{array}\right.
$$

For alternative derivations of the short-time linear response law $\sim t^{3 / 4}$, see Refs. $[8,45,46]$. The crossover time $t_{\mathfrak{f}} \equiv \mathfrak{f}^{-2}$ is the time where the external force $f$ equals the Euler buckling force $\ell_{\perp}^{-2}(t)$ corresponding to the correlation length $\ell_{\perp}(t)$.

These results comprise the predictions of OPT to leading order. As evident from Eq. (24), longitudinal friction forces have thereby been completely neglected, because they are of higher order in $\epsilon$. However, on the semi-infinite arclength interval considered here, a spatially constant change in stored length—no matter how small-implies via Eq. (37) an infinitely fast change $\partial_{t}\left\langle\Delta R_{\|}\right\rangle$of the longitudinal extension and thus friction. Hence, OPT must fail on sufficiently large length scales. Let us define $L_{\star}(t)$ as the length scale beyond which OPT breaks down for a given time $t$. In the present case of a suddenly applied pulling force, this critical length can be estimated from the physical requirement that the total longitudinal friction be not only finite but at most equal to the driving force $\mathfrak{f}-$ i.e.,

$$
\mathfrak{f} \stackrel{!}{=} \hat{\zeta} L \partial_{t}\left\langle\Delta R_{\|}\right\rangle=-\hat{\zeta} L \int_{0}^{L} d s \partial_{t}\langle\Delta \varrho\rangle(s, t) .
$$

For an average stored length given by the spatially constant value, Eq. (53), this condition is met if the polymer length is smaller than

$$
L_{\star}(t) \equiv \sqrt{\mathfrak{f} /\left(\hat{\zeta} \partial_{t}\langle\Delta \bar{\varrho}\rangle\right)} \simeq \begin{cases}\sqrt{\ell_{p} / \hat{\zeta}} t^{1 / 8}, & \text { for } t \ll t_{\mathfrak{f}}, \\ \sqrt{\ell_{p} / \hat{\zeta}}(\mathfrak{f} t)^{1 / 4}, & \text { for } t \gg t_{\mathfrak{f}} .\end{cases}
$$

OPT can thus only be valid on length scales (much) smaller than $L_{\star}(t)$. The condition 


$$
L_{\star}\left(t_{\star}\right)=L
$$

implicitly defines the critical time scale $t_{\star}$ above which OPT applies to the whole polymer and below which OPT is limited to subsections shorter than $L_{\star}(t)$.

In summary, the omission of tension propagation has been identified as the reason for the breakdown of the OPT predictions. On a heuristic level [6], the problem can thus be resolved by requiring that the applied tension be not immediately perceptible everywhere in the filament, but propagate a finite distance $\ell_{\|}(t)$ during time $t$ from the ends towards the bulk of the filament. Hence, only segments up to a distance $\ell_{\|}(t)$ from the ends are set into longitudinal motion. If the length $\ell_{\|}(t)$ over which the tension varies is smaller than the critical length $L_{\star}(t)$, it is ensured that the longitudinal friction does not exceed the driving force. In heuristic discussions, it was generally assumed that both lengths can be identified up to numerical factors of order 1 ,

$$
\ell_{\|}(t) \simeq L_{\star}(t), \quad t \ll t_{\star} \quad \text { (heuristic hypothesis) }
$$

as summarized in Table I, right. The scaling assumption in Eq. (57) turns out to reproduce the correct scaling for most cases (with, however, interesting exceptions elaborated in part II [10], as well as, in Ref. [12]). The "weak"- and strong"-force limits $\ell_{\|} \propto t^{1 / 8}$ and $\ell_{\|} \propto t^{1 / 4}$ of Refs. [4,6] are with Eqs. (55) and (57) recovered as "short"- and long"-time asymptotics. The crossover at time $t_{\mathfrak{f}}$ signals the change from "free" to "forced" relaxation and is inherited from the one of the scaling function $\Sigma$, defined in Eq. (50).

Finally, we note that the tension may be considered as "slowly" varying in space because $\ell_{\|}(t) \propto O\left(\epsilon^{-1 / 2}\right)$ is for $\epsilon$ $\rightarrow 0$ larger than any length that does not dependent on the small parameter (for $t, \mathfrak{f}$, and $L$ fixed). As it turns out, the most important length of the latter type is the dynamic correlation length $\ell_{\perp}(t)$ for transverse displacements. Namely, the scale separation

$$
\ell_{\|} / \ell_{\perp}=O\left(\epsilon^{-1 / 2}\right) \gg 1
$$

indicates that the tension is nearly constant on the equilibration length scale $\ell_{\perp}(t)$ for transverse displacements. Intuitively, it should be clear that this simplifies the further analysis considerably, because it allows one to apply (certain) results locally that are derived for spatially constant tension. Formally, Eq. (58) lends itself as a starting point for a multiple-scale calculus, which separates the physics on different scales to obtain an improved perturbation expansion that is regular in the limit $t \rightarrow 0$ while $\epsilon \ll 1$ is fixed. ${ }^{6}$ The

\footnotetext{
${ }^{6}$ It may be remarked that the encountered contradiction does not appear if one takes the limit $\epsilon \rightarrow 0$ while the parameters $L$ and $t$ are held fixed. This corresponds to a lower temperature and a stiffer polymer, respectively. Then, the physically motivated requirement of the external force exceeding the total longitudinal friction force is satisfied for small enough $\epsilon \ll 1$. The same conclusion may be drawn from the crossover length scales $L_{\star}$ in Eq. (55) upon eliminating $\ell_{p}$ in favor of the small parameter $\epsilon$, because $L_{\star}(\epsilon, t)>L$ for given $L$ and $t$ and small enough $\epsilon$. In a mathematical sense, the expansion generated by ordinary perturbation is pointwise
}

procedure, detailed in the next section, is similar in spirit to the procedure for athermal rod dynamics [47], but some complications related to the stochastic nature of the equations of motion have to be faced. The final result will be an effective deterministic description of the tension on the macroscale $\ell_{\|}(t)$, where the stochasticity on the microscale $\ell_{\perp}(t)$ has been integrated out.

\section{MULTIPLE-SCALE ANALYSIS}

We introduce a rapidly and a slowly varying arclength coordinate, $x \equiv s$ and $y \equiv s \epsilon^{\gamma}$, respectively, where the exponent $\gamma>0$ will be fixed later. The dynamic functions $\boldsymbol{r}_{\perp}$ and $f$ are now considered to depend on both variables $\left\{f, \boldsymbol{r}_{\perp}\right\}$ $\rightarrow\left\{f(x, y), \boldsymbol{r}_{\perp}(x, y)\right\}$, where $x$ and $y$ are treated as independent. The original arclength derivative of those functions then becomes

$$
\left.\partial_{s} \equiv \partial_{x}\right|_{y}+\left.\epsilon^{\gamma} \partial_{y}\right|_{x}
$$

The noise $\xi=O\left(\epsilon^{1 / 2}\right)$ being the source of any transverse displacements suggests an expansions of the dynamic variables $\boldsymbol{r}_{\perp}$ and $f$ in powers of $\epsilon^{1 / 2}$,

$$
\begin{gathered}
\boldsymbol{r}_{\perp}(x, y)=\boldsymbol{\epsilon}^{1 / 2} \boldsymbol{h}_{1}(x, y)+o\left(\boldsymbol{\epsilon}^{1 / 2}\right), \\
f(x, y)=f_{0}(x, y)+\epsilon^{1 / 2} f_{1}(x, y)+\epsilon f_{2}(x, y)+o(\boldsymbol{\epsilon}) .
\end{gathered}
$$

In the case of isotropic friction (i.e., $\zeta_{\perp}=\zeta_{\|}$), the stochastic forces have no intrinsic scale. Hence, they can only depend on the microscopic variable, $\boldsymbol{\xi}=\boldsymbol{\epsilon}^{1 / 2} \boldsymbol{\xi}_{1}(x)$. In the anisotropic case, the friction forces and, hence, the stochastic forces are coupled to the orientation of the filament, so that one has to assume a power expansion

$$
\begin{gathered}
\xi_{\perp}(x, y)=\epsilon^{1 / 2} \xi_{\perp, 1}(x)+o\left(\epsilon^{1 / 2}\right), \\
\xi_{\|}(x, y)=\epsilon^{1 / 2} \xi_{\|, 1}(x)+\epsilon \xi_{\|, 2}(x, y)+o(\epsilon) .
\end{gathered}
$$

The $y$ arguments in Eq. (61) are inherited from the $y$ arguments of $\boldsymbol{r}^{\prime}$ entering the noise correlations in Eq. (10b) via the friction matrix, defined in Eq. (8). The $y$ dependence would disappear for isotropic friction. Note that the leading order $\boldsymbol{\xi}_{1}(x)$ still depends on the microscopic variable $x$ only, because the anisotropy merely enters the higher orders (a formal argument is given in Appendix B 1).

In the following, it is crucial to require that the expansion coefficients in each order be bounded, so that we obtain a uniformly valid power expansion [48] in terms of the small parameter $\epsilon$. Inserting all expansions, Eqs. (61) and (60), into the equations of motion, Eqs. (19a) and (19b), yields

$$
0=\epsilon^{1 / 2}\left[\partial_{t} \boldsymbol{h}_{1}+\partial_{x}^{4} \boldsymbol{h}_{1}-\partial_{x}\left(f_{0} \partial_{x} \boldsymbol{h}_{1}\right)-\boldsymbol{\xi}_{\perp, 1}\right]+o\left(\epsilon^{1 / 2}\right)
$$

asymptotic in $t$, but not uniformly [52]: the smaller $t$ the smaller $\epsilon$ has to be for the expansion to be asymptotic. 


$$
\begin{aligned}
0= & \partial_{x}^{2} f_{0}+\epsilon^{\gamma} 2 \partial_{x} \partial_{y} f_{0}+\epsilon^{1 / 2}\left[\partial_{x}^{2} f_{1}+\xi_{\|, 1}^{\prime}\right]+\epsilon^{2 \gamma} \partial_{y}^{2} f_{0}+\epsilon\left[\partial_{x}^{2} f_{2}\right. \\
& \left.+X_{2}(x, y)+\xi_{\|, 2}^{\prime}\right]+\epsilon^{\gamma+1 / 2} \partial_{y} \partial_{x} f_{1}+o\left(\epsilon ; \epsilon^{2 \gamma}\right) .
\end{aligned}
$$

In order to arrive at Eq. (62b) we used the local arclength constraint, Eq. (15). By

$$
\begin{aligned}
X_{2}(x, y)= & \frac{\hat{\zeta}}{2} \partial_{t}\left(\partial_{x} \boldsymbol{h}_{1}\right)^{2}+(1-\hat{\zeta}) \partial_{x}\left[\left(\partial_{x} \boldsymbol{h}_{1}\right)\left(\partial_{t} \boldsymbol{h}_{1}\right)\right]+\frac{1}{2} \partial_{x}^{4}\left(\partial_{x} \boldsymbol{h}_{1}\right)^{2} \\
& -\frac{1}{2} \partial_{x}^{2}\left[f_{0}\left(\partial_{x} \boldsymbol{h}_{1}\right)^{2}\right]
\end{aligned}
$$

we have summarized terms nonlinear in $\boldsymbol{h}_{1}$. The first term in $X_{2}$, which is proportional to $\hat{\zeta}$, accounts for the longitudinal friction and is thus responsible for the short-time divergence encountered in the heuristic discussion of Sec. IV.

The $O(1)$ part of Eq. (62b), $\partial_{x}^{2} f_{0}=0$, together with the requirement of $f_{0}$ being bounded for large $x$, implies that

$$
f_{0}(x, y)=\hat{f}_{0}(y)
$$

is independent of $x$. Hence both the $O(1)$ and $O\left(\epsilon^{\gamma}\right)$ term of Eq. (62b) vanish. Requiring the $O\left(\epsilon^{1 / 2}\right)$ coefficient to be zero fixes $f_{1}$ up to an integration constant. The value of $f_{1}$ does not affect the evolution of $\boldsymbol{h}_{1}$ because it does not enter the $O(\epsilon)$ coefficient in Eq. (62a). Thus, the precise value of $f_{1}$ does not change the pathological behavior of longitudinal friction $\propto \hat{\zeta} \partial_{t}\left(\partial_{x} \boldsymbol{h}_{1}\right)^{2}$, which is why we shift the discussion of $f_{1}$ to Appendix B 2. From the latter we merely need that $\partial_{y} \partial_{x} f_{1}(x, y)=0$ which renders the $O\left(\epsilon^{\gamma+1 / 2}\right)$ term in Eq. (62b) zero.Then the next higher order is either $O\left(\epsilon^{2 \gamma}\right)$ or $O(\epsilon)$ depending on the value of $\gamma$. With Eq. (64) we can solve the $O\left(\epsilon^{1 / 2}\right)$ part of Eq. (62a) for $\boldsymbol{h}_{1}(x, y)$ along the lines of Sec. III A and use the result to evaluate $X_{2}(x, y)$. It then turns out that the first term in $X_{2}$ (the longitudinal friction) would require $f_{2}$ to grow without bound with increasing system size to render the $O(\epsilon)$-expansion coefficient in Eq. (62b) finite, if they were required to balance each other. This represents the same unphysical divergence that is responsible for the breakdown of ordinary perturbation theory, discussed in Sec. IV.

In order to obtain an improved perturbation theory, we attempt to balance the nonlinear term by the $O\left(\epsilon^{2 \gamma}\right)$ term after choosing $\gamma=1 / 2$; i.e., the exponent $\gamma$ is fixed such that the expansion coefficient $f_{2}$ remains finite in the semi-infinite system considered here. ${ }^{7}$ The equation fixing $f_{2}$ thus reads

$$
\partial_{x}^{2} f_{2}(x, y)=-\partial_{y}^{2} \hat{f}_{0}(y)-X_{2}(x, y)-\xi_{\|, 2}^{\prime}
$$

Given $h_{1}$ and $f_{0}$ the last equation can be solved for $f_{2}$ :

\footnotetext{
${ }^{7}$ We note aside that the small parameter $\epsilon^{\gamma}=\epsilon^{1 / 2}$ appearing here is the same as in the length scale separation, Eq. (58), observed in Sec. IV.
}

$$
f_{2}(x, y)=\int_{0}^{x} d \widetilde{x} \int_{0}^{\tilde{x}} d \hat{x}\left\{-\partial_{y}^{2} \hat{f}_{0}(y)-X_{2}(\hat{x}, y)-\xi_{\|, 2}^{\prime}(\hat{x})\right\} .
$$

For $f_{2}$ to be bounded for large system sizes, we have to require

$$
\partial_{y}^{2} \hat{f}_{0}(y)={\overline{-X_{2}-\xi_{\|, 2}^{\prime}}}^{x}(y),
$$

where the overbar denotes the spatial average over the rapidly varying coordinate $x$,

$$
\bar{g}^{\mathrm{x}}(y) \equiv \lim _{l \rightarrow \infty} \int_{0}^{l} \frac{d x}{l} g(x, y),
$$

for a function $g(x, y)$. The expansion coefficient $f_{2}$ would show a divergence quadratic in the system size if Eq. (67) was not satisfied. Hence, the $y$ dependence of $\hat{f}_{0}(y)$ must be fixed such that the expansion coefficient $f_{2}$ remains finite. For a finite polymer, the limit $l \rightarrow \infty$ is not to be taken literally, though. Rather, the average in Eq. (68) is required to become independent of $l$ to leading order in $\epsilon$ for $l$ much smaller than the system size $L$.

As it turns out, the only quantity in Eq. (67) that does not vanish upon $x$ averaging is the first term in $X_{2}$, the longitudinal friction. This is easily seen for all other terms in $X_{2}$ and the $f_{1}$ term. They are total derivatives with respect to $x$ of products of expansion coefficients that are (required to remain) bounded (nonsecular [48]) by definition. Hence, the $x$ integrals of those total derivatives are bounded and the $x$ averages vanish upon formally taking the coarse-graining length $l \rightarrow \infty$ in Eq. (68). The noise term also represents a total derivative with respect to $x$. The average of that term represents a stochastic variable with an amplitude that scales as $1 / l$ and, hence, also vanishes in the limit $l \rightarrow \infty$.

Dropping all terms that vanish under coarse graining and integrating over time, Eq. (67) takes the form

$$
\frac{1}{\hat{\zeta}} \partial_{y}^{2} \hat{F}_{0}(y)=\frac{1}{2}\left[{\overline{\left(\partial_{x} \boldsymbol{h}_{1}\right)^{2}}}^{x}(y, 0)-{\overline{\left(\partial_{x} \boldsymbol{h}_{1}\right)^{2}}}^{x}(y, t)\right]=-\epsilon^{-1} \overrightarrow{\Delta \varrho}^{s}(t),
$$

where $\Delta \varrho(s, t)$ is the change in stored length, as defined in Eq. (36), of a semi-infinite polymer for the tension history

$$
F(t)=\hat{F}_{0}(y, t) .
$$

Note that the dependence on the slowly varying arclength coordinate $y$ enters the tension history in Eq. (70) only parametrically. The same holds true for the calculation of the right-hand side of Eq. (69).

The closed set of equations Eqs. (69) and (62a) represents the lowest order of the multiple-scale perturbation expansion. It incorporates the feedback mechanism already found in the heuristic discussion above. The evolution of transverse displacements implies longitudinal motion via the arclength constraint, and according to Eq. (69), the corresponding longitudinal friction sets the polymer under tension. This, in 
turn, feeds back onto the evolution of transverse displacements, Eq. (62a), typically acting as to reduce the longitudinal friction.

For solving Eqs. (69) and (62a) self-consistently, it would be handy to perform an ensemble average $\langle\cdots\rangle$ on the righthand side of Eq. (69), because we could then apply expression (42) for $\langle\Delta \varrho\rangle$. We argue that such an ensemble average is indeed justified, because $\overline{\Delta \varrho}^{s}(t)$ is, in fact, a deterministic quantity as a consequence of the central limit theorem. Recall that the quantity $\langle\Delta \varrho\rangle(s, t)$ is dominated by transverse modes of wavelength $\ell_{\perp}(t)$ given by Eq. (46). Since wavelengths much larger than $\ell_{\perp}(t)$ are not relevant in the mode sum, the dynamic length $\ell_{\perp}(t)$ can be interpreted as the correlation length of the change in stored length-i.e., the length over which the correlation function $C_{s, t}(z)$ $\equiv\langle\Delta \varrho(s, t) \Delta \varrho(s+z, t)\rangle$ varies. Hence, the integral $X_{l}$ $\equiv \int_{d s}^{0} l \Delta \varrho(s, t)$ may be understood as the sum of $l / \ell_{\perp}(t)$ weakly correlated random variables. For large $l$, the distribution function of $X_{l}$ thus becomes Gaussian and the variance of $X_{l}$ grows linearly with the number of independent random variables, $\left\langle\left(X_{l}-\left\langle X_{l}\right\rangle\right)^{2}\right\rangle \propto l$. As a consequence, the distribution of the average $X_{l} / l \rightarrow \overline{\Delta \varrho}^{s}$ approaches a $\delta$ function as $l \rightarrow \infty$.

An "additional" ensemble average therefore does not change the value of the right-hand side of Eq. (69). Evaluating the spatial average after the ensemble average levels out the boundary term of expression (42) for $\left\langle\Delta \varrho_{h / c}\right\rangle$ and reduces it to its bulk value $\langle\Delta \bar{\varrho}\rangle(t)$, defined in Eq. (47), which is completely independent of the boundary conditions. We thus have the important relation

$$
\overrightarrow{\Delta \varrho_{h / c}}(t)=\overrightarrow{\left\langle\Delta \varrho_{h / c}\right\rangle}(t)=\langle\Delta \bar{\varrho}\rangle(t) .
$$

We would like to emphasize that our argument for replacing the spatial by an ensemble average requires a finite driving force, such that $\lim _{l \rightarrow \infty} \overline{\Delta \varrho}^{s}$ approaches a finite value. This specifically excludes the linear response limit-i.e., the limit of vanishing external force $\mathfrak{f} \rightarrow 0$ while $\epsilon \ll 1$ is fixed. In this case, the tension dynamics has to be described by Eq. (69), which is stochastic even to leading order (we will come back to this point in part II)

Given the external driving is finite such that Eq. (71) may be applied, Eq. (69) takes the form

$$
\partial_{s}^{2} F(s, t)=-\hat{\zeta}\langle\Delta \bar{\varrho}\rangle[F(s, \tilde{t} \leq t), t],
$$

where we introduced $s=y \epsilon^{1 / 2}=x$ again and made the parametric dependence of $\langle\Delta \bar{\varrho}\rangle$ on the tension history explicit. The deterministic tension dynamics, as described by Eq. (72), provides the sought-after rigorous local generalization of the heuristic argumentation of Sec. III B: local longitudinal motion is driven by tension gradients (like in a thread pulled through a viscous medium).

Upon inserting our result in Eq. (47) into the right-hand side, we obtain a nonlinear, PIDE for the tension history $F(s, t)$,

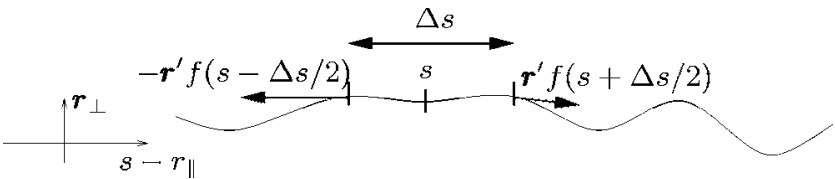

FIG. 4. Tensile forces acting on a polymer subsection of size $\Delta s$. Balancing these forces with the drag that arises from the longitudinal velocity of magnitude $\partial_{t} r_{\|}(s, t)$, one estimates $\Delta s \zeta_{\|} \partial_{t} r_{\|}(s, t)$ $\approx f(s-\Delta s / 2)-f(s+\Delta s / 2)$ in the weakly bending limit. For infinitesimal $\Delta s$, this becomes $\zeta_{\|} \partial_{t} r_{\|}(s, t) \approx-\partial_{s} f(s, t)$. A further spatial derivative yields $\zeta_{\|} \partial_{t} \partial_{s} r_{\|}(s, t) \approx-\partial_{s}^{2} f(s, t)$, which is the time derivative of Eq. (72) (in original units) up to a spatial and ensemble average on the left-hand side, which correspond to an adiabatic and equilibrium approximation, respectively.

$$
\begin{aligned}
\partial_{s}^{2} F(s, t)= & \hat{\zeta} \int_{0}^{\infty} \frac{d q}{\pi \ell_{p}}\left\{\frac{1}{q^{2}+f_{<}}\left(1-e^{-2 q^{2}\left[q^{2} t+F(s, t)\right]}\right)\right. \\
& \left.-2 \theta q^{2} \int_{0}^{t} d \widetilde{t} e^{-2 q^{2}\left[q^{2}(t-\widetilde{t})+F(s, t)-F(s, \tilde{t}]\right]}\right\} .
\end{aligned}
$$

We have arrived at a closed description of the polymer dynamics to lowest order in MSPT that consists of two parts. On a length scale $\ell_{\perp}(t)$, Brownian motion gives rise to fluctuations of transverse displacements that are described by the linear Equation (62a). This stochastic differential equation, in turn, adiabatically depends on a tension profile that varies on a much larger scale $\ell_{\|}(t)$ and satisfies a deterministic nonlinear equation of motion, Eq. (73).

\section{CONCLUSION}

We would like to conclude the present general discussion of tension dynamics with a simple physical interpretation of the outcome, Eqs. (72) and (73), of our multiple-scale analysis. Effectively, our MSPT analysis is a rigorous justification of certain approximations that can be made to analyze the tension dynamics in the small- $\epsilon$ limit. According to Eq. (72), the curvature of the integrated tension is (up to a constant) given by the ensemble average of the local stored length release. As shown in Fig. 4, it may be conceived as (i) a force balance equation between the locally acting tensile and longitudinal friction force, in which (ii) the latter may be computed from the equations of motion for the bulk of an equilibrated polymer under a spatially constant, though timedependent, tension. The longitudinal friction force is obtained via taking a time derivative of this coarse-grained dynamical force extension relation, Eq. (47). The first-order MSPT equation of motion neglects longitudinally acting bending forces, employs an adiabatic approximation, and assumes local equilibrium. The latter fully retains memory effects and therefore must not be mistaken as an approximation of quasistationary dynamics, which, in part II [10], will turn out to be a valid approximation only for specific driving forces in a particular time regime.

\section{ACKNOWLEDGMENTS}

It is a pleasure to acknowledge helpful conversations with 
Benedikt Obermayer. This research was supported by the German Academic Research Foundation (DAAD) through a grant within the Postdoc-Program $(\mathrm{OH})$ and by the Deutsche Forschungsgemeinschaft through Grants Nos. Ha 5163/1 (O.H.) and SFB 486 (E. F.).

\section{APPENDIX A: ELASTIC FORCES}

From the effective free energy of a wormlike chain, Eq. (2), we seek to determine the elastic force $\boldsymbol{g}_{\text {el }}(s)$ per arclength that a polymer of a given conformation $\overline{\boldsymbol{r}}(s)$ exerts at arclength $s$ onto its surroundings. To this end, let us assume the polymer was subject to a constant external force field equal to $-\boldsymbol{g}_{\mathrm{el}}(s)$, the negative of the local elastic forces. Then, the conformation $\overline{\boldsymbol{r}}(s)$ is in balance with the external force and minimizes the total free energy

$$
\mathcal{H}[\boldsymbol{r}]=\mathcal{H}_{\mathrm{WLC}}[\boldsymbol{r}]+\mathcal{H}_{\mathrm{ext}}[\boldsymbol{r}],
$$

with

$$
\mathcal{H}_{\mathrm{ext}}[\boldsymbol{r}] \equiv \int_{0}^{L} d s \boldsymbol{g}_{\mathrm{el}}(s) \boldsymbol{r}(s),
$$

under all possible paths that obey the local inextensibility, Eq. (1). Hence, requiring a vanishing free energy change $\delta \mathcal{H}$ for an infinitesimal (permitted) change $\delta \boldsymbol{r}$ in the space curve leads to the sought-after relation between the elastic forces and the contour. The central question of the minimization problem is how to deal with the local constraint.

Here, we show that the common [49] introduction of a Lagrange multiplier function ensuring the local inextensibility constraint is not necessary. We will instead present a minimization procedure that considers only variations that obey the inextensibility constraint to leading order.

Consider a test contour

$$
\boldsymbol{r}(s)=\overline{\boldsymbol{r}}(s)+\delta \boldsymbol{r}(s),
$$

which is infinitesimally displaced by $\delta \boldsymbol{r}(s)$ from the equilibrium contour $\overline{\boldsymbol{r}}(s)$. The inextensibility constraint, Eq. (1), is fulfilled to $O(\delta r)$ if we only consider displacements that are constructed from another infinitesimal vector field $\boldsymbol{\epsilon}(s)$ according to

$$
\delta \boldsymbol{r}^{\prime}(s)=\boldsymbol{\epsilon}(s) \times \overline{\boldsymbol{r}}^{\prime}(s) .
$$

Those displacements are transverse to the local tangent vector, so that $\delta\left(\boldsymbol{r}^{\prime 2}\right)$ is quadratic in $\delta \boldsymbol{r}$. They correspond to local rotations of the tangents.

The variation of the contour induces a variation $\delta \mathcal{H}$ of the total elastic energy $\mathcal{H}=\mathcal{H}_{\mathrm{wLC}}+\mathcal{H}_{\text {ext }}$ of the form

$$
\begin{aligned}
\delta \mathcal{H} & =\int_{0}^{L} d s\left(\kappa \overrightarrow{\boldsymbol{r}}^{\prime \prime} \delta \boldsymbol{r}^{\prime \prime}+\boldsymbol{g}_{\mathrm{el}} \delta \boldsymbol{r}\right) \\
& =-\int_{0}^{L} d s\left(\kappa \overrightarrow{\boldsymbol{r}}^{\prime \prime \prime}+\int_{0}^{s} d \widetilde{s} \boldsymbol{g}_{\mathrm{el}}\right) \delta \boldsymbol{r}^{\prime}+\mathrm{b} \cdot \mathrm{t} .
\end{aligned}
$$

$$
=-\int_{0}^{L} d s\left(\kappa \overrightarrow{\boldsymbol{r}}^{\prime \prime}+\boldsymbol{f}_{\mathrm{el}}\right)\left(\boldsymbol{\epsilon} \times \overline{\boldsymbol{r}}^{\prime}\right)+\mathrm{b} \cdot \mathrm{t} .
$$

$$
=-\int_{0}^{L} d s \epsilon\left[\overline{\boldsymbol{r}}^{\prime} \times\left(\kappa \overrightarrow{\boldsymbol{r}}^{\prime \prime \prime}+f_{\mathrm{el}}\right)\right]+\mathrm{b} \cdot \mathrm{t} .
$$

Here, we performed a partial integration to obtain Eq. (A5b), which introduces some boundary terms abbreviated by "b.t." In the subsequent step we inserted Eq. (A4) for $\delta \boldsymbol{r}^{\prime}$ and introduced the arclength-dependent force

$$
\boldsymbol{f}_{\mathrm{el}}(s) \equiv \int_{0}^{s} d \widetilde{s} \boldsymbol{g}_{\mathrm{el}}(\widetilde{s}) .
$$

Finally, we used the property $\boldsymbol{A} \cdot(\boldsymbol{B} \times \boldsymbol{C})=\boldsymbol{C} \cdot(\boldsymbol{A} \times \boldsymbol{B})$ of the triple scalar product to obtain Eq. (A5d).

If $\overline{\boldsymbol{r}}(s)$ is indeed the equilibrium contour, then $\delta \mathcal{H}$ has to vanish for all variations parametrized by $\boldsymbol{\epsilon}(s)$ and $\delta \boldsymbol{r}(L)$. Therefore, the term in the square brackets of the integrand in Eq. (A5d) has to vanish,

$$
\overline{\boldsymbol{r}}^{\prime} \times\left(\boldsymbol{\kappa} \overrightarrow{\boldsymbol{r}}^{\prime \prime}+f_{\mathrm{el}}\right)=0,
$$

so that we recover Eq. (4), used in Sec. II. In addition, the boundary terms

$$
\begin{aligned}
\mathrm{b} . \mathrm{t} .= & \kappa\left(\overrightarrow{\boldsymbol{r}}^{\prime \prime} \delta \boldsymbol{r}^{\prime}\right)\left|{ }_{0}^{L}+\delta \boldsymbol{r}(L) \int_{0}^{L} d \widetilde{s} \boldsymbol{g}_{\mathrm{el}}=\kappa\left[\overrightarrow{\boldsymbol{r}}^{\prime \prime}\left(\boldsymbol{\epsilon} \times \overline{\boldsymbol{r}}^{\prime}\right)\right]\right|{ }_{0}^{L} \\
& +\left.\left[\boldsymbol{f}_{\mathrm{el}} \delta \boldsymbol{r}\right]\right|^{L}
\end{aligned}
$$

have to cancel, implying the requirements

$$
\begin{gathered}
\boldsymbol{f}_{\mathrm{el}}(L)=0, \\
\left.\left(\overline{\boldsymbol{r}}^{\prime} \times \overline{\boldsymbol{r}}^{\prime \prime}\right)\right|_{0, L}=0 .
\end{gathered}
$$

The condition expressed by Eq. (A9) simply states that a force balance can only exist if the external forces sum up to zero. Using the inextensibility constraint, Eq. (1), it is seen that $\overrightarrow{\boldsymbol{r}}^{\prime \prime} \cdot \overline{\boldsymbol{r}}^{\prime}=0$, so that Eq. (A10) can be rewritten as the boundary condition

$$
\left.\overrightarrow{\boldsymbol{r}}^{\prime \prime}\right|_{0, L}=0 \text {. }
$$

The curvature, which is proportional to the local torque, has to vanish at the (free) ends.

Note that the overbar in $\overline{\boldsymbol{r}}(s)$ to denote the equilibrium contour is dropped in the main text, for simplicity.

\section{APPENDIX B: MULTIPLE-SCALE ANALYSIS (DETAILS)}

\section{Thermal forces}

In the multiple-scale perturbation theory, presented in Sec. $\mathrm{V}$, one should, in principle, assume an expansion $\boldsymbol{\xi}(x, y ; t)$ $=\epsilon^{1 / 2} \boldsymbol{\xi}_{1}(x, y, t)+o(\epsilon)$ where the leading-order noise $\boldsymbol{\xi}_{1}$ is a function of the coarse-grained variable $y$. However, from the 
fundamental correlations obeyed by the original noise function, Eq. (10b), we have to require to leading order

$$
\left\langle\boldsymbol{\xi}_{1}(s, \epsilon s ; t) \boldsymbol{\xi}_{1}\left(s^{\prime}, \epsilon s^{\prime} ; t\right)\right\rangle=2(\mathbf{I} / L) \delta\left(s-s^{\prime}\right) \delta\left(t-t^{\prime}\right)
$$

for all $\epsilon \equiv L / \ell_{p}$. Apparently, the right-hand side of Eq. (B1) does not depend on $\epsilon$. As a consequence, the left-hand side cannot depend on $\epsilon$ either, in particular not on $\epsilon s^{\prime}$ or $\epsilon s$. Hence, the two-point correlations are independent of the slowly varying arclength coordinate. Using Wick's theorem, we may argue in the same way for all higher-order correlation functions as well and conclude that the leading-order stochastic force $\boldsymbol{\xi}_{1}$ is itself independent of $y$.

We note that the inverse length appearing on the righthand side of Eq. (B1) is due to the definition $\epsilon \equiv L / \ell_{p}$ of the small parameter for a stiff polymer. In the case of a strongly prestretched polymer, the small parameter is defined as $\epsilon$ $\equiv\left(\ell_{p} \mathfrak{f}\right)^{-1 / 2}$ [see Eq. (13) and the subsequent paragraph], so that $L^{-1}$ in Eq. (B1) has to be replaced by $\mathfrak{f}^{-1 / 2}$.

\section{Next to leading order tension}

Requiring the $O\left(\epsilon^{1 / 2}\right)$ coefficient in Eq. (62b) yields a noisy first-order tension

$$
f_{1}(x, y)=\int_{0}^{x} d \widetilde{x}\left[\xi_{\|, 1}(0)-\xi_{\|, 1}(\widetilde{x})\right]+b_{1}(y) x+a_{1}(y) .
$$

For $f_{1}$ to be bounded in $x$, the term $b_{1}(y) x$ has to cancel the linearly growing noise term on the right-hand side,

$$
b_{1}=\lim _{x \rightarrow \infty} \frac{1}{x} \int_{0}^{x} d \widetilde{x}\left[\xi_{\|, 1}(0)-\xi_{\|, 1}(\widetilde{x})\right] .
$$

However, important for the multiple-scale analysis in Sec. V is merely that $b_{1}$ is independent of $y$, so that $\partial_{y} \partial_{x} f_{1}(x, y)=0$.
[1] E. Frey and K. Kroy, Ann. Phys. 14, 20 (2005).

[2] M. L. Gardel, J. H. Shin, F. C. MacKintosh, L. Mahadevan, P. Matsudaira, and D. A. Weitz, Science 304, 1301 (2004).

[3] J. Baschnagel et al., Adv. Polym. Sci. 152, 41 (2000).

[4] U. Seifert, W. Wintz, and P. Nelson, Phys. Rev. Lett. 77, 5389 (1996).

[5] A. Ajdari, F. Jülicher, and A. Maggs, J. Phys. I 7, 823 (1997).

[6] R. Everaers, F. Jülicher, A. Ajdari, and A. C. Maggs, Phys. Rev. Lett. 82, 3717 (1999).

[7] F. Brochard-Wyart, A. Buguin, and P. G. de Gennes, Europhys. Lett. 47, 171 (1999).

[8] D. C. Morse, Macromolecules 31, 7044 (1998).

[9] O. Hallatschek, E. Frey, and K. Kroy, Phys. Rev. Lett. 94, 077804 (2005).

[10] O. Hallatschek, E. Frey, and K. Kroy, following paper, Phys. Rev. E 75, 031906 (2007).

[11] B. Obermayer, O. Hallatschek, E. Frey, and K. Kroy, e-print cond-mat/0603556.

[12] B. Obermayer, K. Kroy, E. Frey, and O. Hallatschek (unpublished).

[13] B. Obermayer and O. Hallatschek (unpublished).

[14] D. R. Nelson, Defects and Geometry in Condensed Matter Physics (Cambridge University Press, Cambridge, England, 2002).

[15] H. Yamakawa, Helical Wormlike Chains in Polymer Solutions (Springer, New York, 1997).

[16] E. Frey, K. Kroy, J. Wilhelm, and E. Sackmann, in Dynamical Networks in Physics and Biology, edited by G. Forgacs and D. Beysens (Springer-Verlag, Berlin, 1998).

[17] N. Saitô, K. Takahashi, and Y. Yunoki, J. Phys. Soc. Jpn. 22, 219 (1967).

[18] T. T. Perkins, D. E. Smith, and S. Chu, Science 276, 2016 (1997).

[19] S. R. Quake, H. Babcock, and S. Chu, Nature (London) 388, 151 (1997)

[20] D. Collin, F. Ritort, C. Jarzynski, S. B. Smith, I. Tinoco, and C. Bustamante, Nature (London) 437, 231 (2005).

[21] G. Forgacs, S. H. Yook, P. A. Janmey, H. Jeong, and C. G.
Burd, J. Cell. Sci. 117, 2769 (2004).

[22] P. A. Janmey and D. A. Weitz, Trends Biochem. Sci. 29, 364 (2004).

[23] L. Le Goff, O. Hallatschek, E. Frey, and F. Amblard, Phys. Rev. Lett. 89, 258101 (2002).

[24] A. Caspi, M. Elbaum, R. Granek, A. Lachish, and D. Zbaida, Phys. Rev. Lett. 80, 1106 (1998).

[25] R. Shusterman, S. Alon, T. Gavrinyov, and O. Krichevsky, Phys. Rev. Lett. 92, 048303 (2004).

[26] M. Hohenadl, T. Storz, H. Kirpal, K. Kroy, and R. Merkel, Biophys. J. 77, 2199 (1999).

[27] G. I. Barenblatt, Scaling, Self-Similarity, and Intermediate Asymptotics (Cambridge University Press, Cambridge, England, 1996).

[28] L.D. Landau and E. M. Lifshitz, Theory of Elasticity, Vol. 7 of Course of Theoretical Physics (Pergamon Press, London, 1959).

[29] J. Deutsch, Science 240, 922 (1988).

[30] M. Doi and S. F. Edwards, The Theory of Polymer Dynamics (Clarendon Press, Oxford, 1986).

[31] G. K. Batchelor, J. Fluid Mech. 44, 419 (1970).

[32] R. G. Cox, J. Fluid Mech. 44, 791 (1970).

[33] R. G. Cox, J. Fluid Mech. 45, 625 (1971).

[34] P. G. de Gennes, Scaling Concepts in Polymer Physics (Cornell University Press, Ithaca, 1979).

[35] M. Toda, R. Kubo, and N. Saito, Statistical Physics I: Equilibrium Statistical Mechanics (Springer, Berlin, 1983).

[36] R. Kubo, M. Toda, and N. Hashitsume, Statistical Physics II: Nonequilibrium Statistical Mechanics (Springer, Berlin, 1992).

[37] M. Fixman, J. Chem. Phys. 69, 1527 (1978).

[38] E. J. Hinch, J. Fluid Mech. 271, 219 (1994).

[39] D. Morse, Adv. Chem. Phys. 128, 65 (2004).

[40] B. Y. Ha and D. Thirumalai, J. Chem. Phys. 106, 4243 (1997).

[41] N. K. Lee and D. Thirumalai, Biophys. J. 86, 2641 (2004).

[42] L. Harnau, R. G. Winkler, and P. Reineker, J. Chem. Phys. 102, 7750 (1995).

[43] J. F. Marko and E. D. Siggia, Macromolecules 28, 8759 (1995). 
[44] C. H. Wiggins, D. Riveline, A. Ott, and R. E. Goldstein, Biophys. J. 74, 1043 (1998).

[45] R. Granek, J. Phys. II 7, 1761 (1997).

[46] F. Gittes and F. C. MacKintosh, Phys. Rev. E 58, R1241 (1998).

[47] O. Hallatschek, E. Frey, and K. Kroy, Phys. Rev. E 70, 031802 (2004).

[48] M. H. Holmes, Introduction to Perturbation Methods (Springer, New York, 1995), Chap. 5.
[49] R. E. Goldstein and S. A. Langer, Phys. Rev. Lett. 75, 1094 (1995).

[50] N. G. van Kampen, Stochastic Processes in Physics and Chemistry (North-Holland, Amsterdam, 1981).

[51] Y. Bohbot-Raviv, W. Z. Zhao, M. Feingold, C. H. Wiggins, and R. Granek, Phys. Rev. Lett. 92, 098101 (2004).

[52] E. J. Hinch, Perturbation Methods (Cambridge University Press, Cambridge, England, 1991). 